\title{
Methods and means of increasing operation efficiency of the fleet of electric motors in non-ferrous metallurgy
}

\author{
S. P. Kurilin, Professor, Department of Electromechanical Systems ${ }^{1}$, e-mail: sergkurilin@gmail.com \\ M. I. Dli, Professor, Head of the Department of Information Technologies in Economics and Management ${ }^{1}$, \\ e-mail: midli@mail.ru \\ Y. B. Rubin, Professor, Head of the Department of Theory and Practice of Competition ${ }^{3}$ \\ M. V. Chernovalova, Post-Graduate Student ${ }^{2}$, e-mail: 0208margarita@bk.ru \\ ${ }^{1}$ Branch of the National Research University "Moscow Power Engineering Institute", Smolensk, Russia. \\ 2 National Research University "Moscow Power Engineering Institute", Moscow, Russia. \\ ${ }^{3}$ Moscow University for Industry and Finance "Synergy", Moscow, Russia.
}

\begin{abstract}
Arranging efficient operation of the fleet of induction motors (IM) in non-ferrous metallurgy is a large-scale technical and economic problem. In scientific aspect, the problem is being solved in the framework of two research lines: in developing criteria for the efficient operation of the branch IM fleet and towards the development of methods and tools for implementing the IM fleet efficient operation. The article presents the results of the authors' work in the mentiond areas.

The basis for developing criteria for efficient operation is modeling of current operational states, taking into account the IM operational aging processes. The existing methods and models are poorly focused on fixation of the changes caused by operational aging. There exists a demand for special methods and tools for modeling the IM operational conditions. A mathematical model based on Kolmogorov equations is one of these tools. The system graph and equations of the mathematical model are given. An example of a practical calculation of the no-failure operation probabilities at different rates of repair operations is given. It is stated that the offered mathematical model can serve as an instrument for developing criteria of the IM pool efficient operation.

The system of periodic operational diagnostics is ment to be a key element in enhancement of the IM fleet operation efficiency. A topological method worked out for the problems of operational diagnostics is focused upon analyzing the dynamics of operational changes taking place in the IM vector space. The matrix of current deviations is a medium of objective and reliable information about the current IM technical condition. Matching the matrices of current and limiting deviations allows us to make several essential conclusions concerning the IM technical state.
\end{abstract}

Key words: induction motor, operational aging, efficient operation, reliability, Kolmogorov equations, topological method, periodic operational diagnostics.

DOI: $10.17580 / \mathrm{nfm} .2020 .02 .09$

\section{Introduction}

$\Delta$ high level of electric energy consumption is characteristic of non-ferrous metallurgy enterprises. A typical example is the RUSAL Bratsk public company, which consumes about $75 \%$ of electric power generated by the Bratsk hydroelectric power station. One of the reasons for this is the high-powered electrothermal and electrochemical plants used in non-ferrous metallurgy and the other one is the industrial high-powered electrical equipment, that includes electric motors. In general, the metallurgical industry consumes 143 billion $\mathrm{kW} \cdot \mathrm{h}$ of electrical energy per year, which amounts to $13.3 \%$ of its consumption in the Russian Federation.

A significant part of the branch's annual consumption, up to $50-60 \%$, falls to the share of electric drives of metallurgical industry. If annual consumption of electrical energy by electric drives in metallurgy may be estimated as 70 billion $\mathrm{kWh}$, then their total installed capacity will be 8 million $\mathrm{kW}$. This is equivalent to a pool of one million of a 7-8 kW induction motors operating continuously for a year. This assessment of the branch pool is cited taking into account that the overwhelming majority of electric drives in metallurgy are asynchronous and are based on both specialized electric motors, and the general-purpose ones. At that, the total current cost of electric motors in the branch fleet is assessed at 16 billion rubles, which naturally heighten the need for of its efficient operation.

Managing efficient operation of the induction motors (IM) fleet is an important technical and economic problem that can be posed and solved both at the branch level and at the level of individual corporations and large enterprises.

\section{Scientific aspects of the problem}

In the scientific aspect, the accentuated problem puts forward a number of tasks that are within the framework of the following two scientific directions or enlarged scientific problems. 
1. Elaboration of criteria for efficient operation of the sectoral IM fleet hereinafter referred to as "Task 1".

2. Devising the methods and means for implementing the IM fleet efficient operation, hereinafter referred to as "Task 2".

Task 1 and Task 2 differ not only in their principal purpose, but also in their methodology. The investigation into the area of Task 1 is based on statistical information and mathematical modelling of the IM operational states. Research in the field of Task 2 is based on operational information about the current technical condition and the risk of continuing operation of each IM in the fleet. At the same time, such a separation is conditional, and the mentioned methodologies can be considered complementary. The correlated existence of both problems in the context of a common issue suggests that the operational information of Task 2 can be used as corrective statistical information for Task 1 . The converse proposition is also correct: the criteria developed in the framework of Task 1 are the target functions for Task 2.

This article presents some results of the authors' work on the above-mentioned enlarged problems.

\section{Development of criteria for efficient operation of the IM fleet}

The elaboration of criteria for efficient operation is based on modeling of the current operating IM states, followed by a technical and economic evaluation of the condition of the entire fleet of electric motors. At this, in the light of in-service time, modeling of operational conditions have to be performed taking into account the IM operational aging processes. Aging processes directly affect the maintainability parameters. Therefore, the term «efficient operation» implies not only energy efficiency, but also high reliability indicators of IM. This article presents the development of modeling methods and aids for the IM operational states, focused on the preliminary solution of Task 1. Subsequently, it is expected to be adjusted based on the operational testing database of Task 2.

Modeling of the IM operational states is a new line of electromechanics. For a long time, these issues have been accessory on developing methods and tools for operational diagnostics of IM. In such developments, high emphasis has been placed on the methods and tools of diagnosing, while modeling methods and aids were in the background or were designated conceptually. In accordance with the diagnosis methodology, the existing methods can be divided into the methods of physical measurements [1-7] and methods of statistical observations [8-9]. Apprising both of the above-listed groups of methods, it should be noted that they focus on fixing the external manifestations and results of operational aging. At the same time, they are poorly oriented to internal processes that cause these manifestations and results. Meanwhile, the tasks of organizing efficient operation require information not so much about the external manifestations of operational aging, but about the dynamics of internal processes in IM.
Assessing the state of the issue as a whole, the demand for the methods and aids of modeling the IM operational conditions in the context of the stated problem should be mentioned.

The development presented in the article is based on the equations of A. Kolmogorov [10-11]. Their use has the advantage that statistics on individual IM nodes are known. In particular, relative failure rates are published in the reference literature [12], and relative repair rates are chosen by maintenance crew. Besides, as noted above, the source of corrective information on relative failure rates is intended to be the data from the IM operational testing.

The usual application of Kolmogorov equations, to queueing systems for example, gives a result in the form of a probability set of the system states, one of which is the probability of its up state. At that, the probability set expresses the balance between the failure flow and repair flow; when the capacity of both flows is equal, the system stops moving. For systems with operational aging, including IM, achieving a balance is practically impossible due to the continuous change in technical characteristics of the system. For them, stopping movement can be interpreted in the only way - as reaching the limiting state by the system and its withdrawal from service. Therefore, the coefficients of the equations that define the rate of failure and repair flows in such systems play the role of parameters that accelerate or slow down the process of approaching the limiting state. These coefficients are the means of enhancing maintainability at the disposal of maintenance crew; this is primarily related to the frequency of repair operations.

The state graph of the system is shown in Fig. 1. It represents IM as a system consisting of three nodes: stator winding, bearing units, and rotor winding. Here: $S_{0}, S_{1}$, $S_{2}, S_{3}$ are the conditions without failures, with failure of the stator winding, with failure of bearings and with failure of the rotor winding, respectively, $\lambda_{01}, \lambda_{02}, \lambda_{03}$ are the failure rates of corresponding nodes, $\mu_{10}, \mu_{20}, \mu_{30}$ are the repair rates of corresponding nodes.

Kolmogorov equations based on the graph (Fig. 1) for $p_{0}(t), p_{1}(t), p_{2}(t)$, and $p_{3}(t)$ probabilities of the corresponding system states are given below:

$$
\begin{aligned}
& \frac{d p_{0}(t)}{d t}=-\left(\lambda_{01}+\lambda_{02}+\lambda_{03}\right) p_{0}(t)+\mu_{10} p_{1}(t)+\mu_{20} p_{2}(t)+ \\
& +\mu_{30} p_{3}(t) \\
& \frac{d p_{1}(t)}{d t}=\lambda_{01} p_{0}(t)-\mu_{10} p_{1}(t) \\
& \frac{d p_{2}(t)}{d t}=\lambda_{02} p_{0}(t)-\mu_{20} p_{2}(t) \\
& \frac{d p_{3}(t)}{d t}=\lambda_{03} p_{0}(t)-\mu_{30} p_{3}(t)
\end{aligned}
$$

For the initial moment of operation the following equations are valid: 


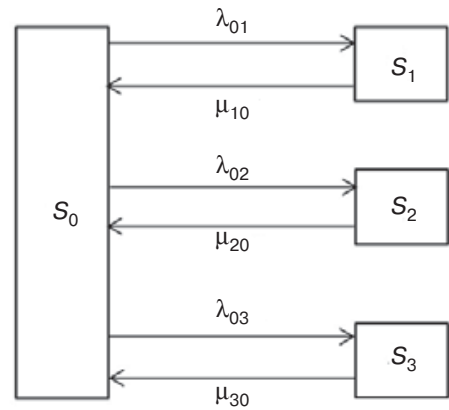

Fig. 1. State graph of the system

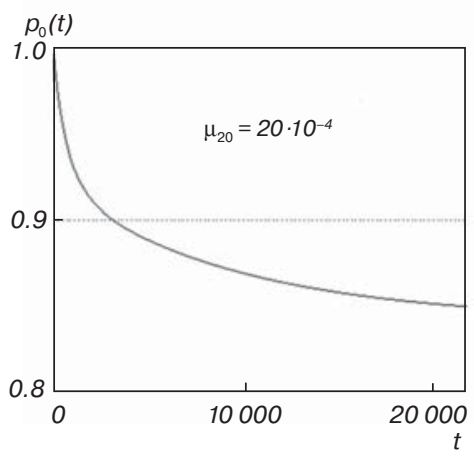

Fig. 2. Probability of the IM survival

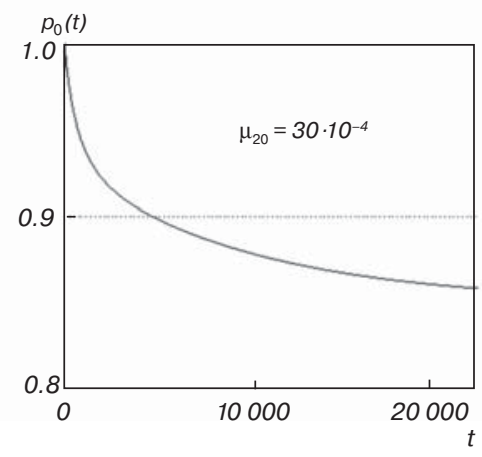

$t$

Table 1

Indicators of the IM maintainability

\begin{tabular}{|l|c|c|}
\hline \multicolumn{1}{|c|}{ Index } & \multicolumn{2}{c|}{ Repair rate of bearing units } \\
\cline { 2 - 3 } & $\mu_{20}=20 \cdot 10^{-4}$ & $\mu_{20}=30 \cdot 10^{-4}$ \\
\hline Probability of no-failure operation at $t=10000$ hours & 0.87 & 0.88 \\
\hline Probability of the stator winding failure at $t=10000$ hours & 0.04 & 0.04 \\
\hline Probability of the bearing failure at $t=10000$ hours & 0.03 & 0.02 \\
\hline Probability of the stator winding failure at $t=10000$ hours & 0.06 & 0.06 \\
\hline Operating time range with a probability of no-failure operation not less than 0.9, hour & $0-3000$ & $0-5000$ \\
\hline Time $T_{r}$ to reach the limiting state, hour & 21000 & 40000 \\
\hline
\end{tabular}

$$
p_{0}(0)=1, p_{1}(0)=0, p_{2}(0)=0, p_{3}(0)=0 \text {. }
$$

The solution of the system (1), (2) carried out for the values of failure rates, nodes per hour ${ }^{-1}$, as follows:

$$
\lambda_{01}=0.5 \cdot 10^{-4}, \lambda_{02}=0.71 \cdot 10^{-4}, \lambda_{03}=0.103 \cdot 10^{-4}
$$

and repair rates, nodes per hour ${ }^{-1}$, as follows:

$$
\mu_{10}=10 \cdot 10^{-4}, \mu_{20}=20 \cdot 10^{-4}, \mu_{30}=1 \cdot 10^{-4}
$$

is depicted as a graph of the $p_{0}(t)$ dependence in Fig. 2. For comparison, the figure also shows the graph of the $p_{0}(t)$ dependence in the case of $\mu_{20}=30 \cdot 10^{-4}$, while preserving the values of other parameter. The dotted line is used for the normalizable probability value of 0.9 .

Comparison of the graphs in Fig. 2 testifies that an increase in the frequency of bearing repairs by 1.5 times has a positive effect on the IM maintainability indexes. Their comparative values are listed in the Table 1.

According to the data provided, an increase of the unit repair rates is an effective means to improve the IM maintainability. Taking into consideration that reliability is a significant component of efficient operation, one can ascertain the following: the presented mathematical model can serve as a means for developing criteria of the IM fleet efficient operation for non-ferrous metallurgy or its individual corporations and enterprises.

\section{Development of methods and implementers for efficient operation of the IM fleet}

The strategy of efficient operation of the sectoral IM fleet should be based on systematic diagnostics of its technical condition. To implement the strategy, operational information about the values of indicators of the technical condition of electric motors is required. Therefore, the system of periodic operational diagnostics is intended to be a key link in enhancement of efficiency of the IM fleet operation.

A topological method was specially developed for operational diagnostics [13-15]. It concentrates on analyzing the dynamics of operational changes occurring in the IM vector space. Within its context, a mathematical model of IM was developed for Task 2, the scientific basis of diagnostics was developed, and methods for assessing the IM operational risks were elaborated. Moreover, the IM vector spaces have been studied and general methodology of their diagnostic testing was defined. The methodology for the IM diagnostic testing is presented below.

In accordance with the topological method, the impulse voltage vectors are oriented during diagnostic testing in three directions $x, y, z$ of the working area of the vector space

$$
u_{S x}=\left(\begin{array}{c}
\delta(t) \\
-\delta(t) \\
0
\end{array}\right), u_{S y}=\left(\begin{array}{c}
0 \\
\delta(t) \\
-\delta(t)
\end{array}\right), u_{S z}=\left(\begin{array}{c}
-\delta(t) \\
0 \\
\delta(t)
\end{array}\right),
$$

where $\delta(t)$ is the Dirac function.

The IM responses the impulse voltage vectors action are three phase current vectors

$$
i_{S x}=\left(\begin{array}{r}
i_{A} \\
-i_{A} \\
i_{C}
\end{array}\right), \quad i_{S y}=\left(\begin{array}{r}
i_{A} \\
i_{B} \\
-i_{B}
\end{array}\right), \quad i_{S z}=\left(\begin{array}{c}
-i_{C} \\
i_{B} \\
i_{C}
\end{array}\right),
$$

containing information about the current state of working area. In particular, the disproportionality of the coordinates 
of similar vectors, for example the $u_{S x}$ and $i_{S x}$ ones, is an evidence of the parametric heterogeneity of the working area and the partial exit of the $i_{S x}$ vector outside its limits. Changes in $i_{S x}, i_{S y}$, and $i_{S z}$ signals recorded over the course of operation indicate changes in the parametric, physical, and technical properties of IM as a result of operational damages and aging.

The current information that is topical for a fixed point in operation time $t$ is stored in the Green's matrix. However, for operational diagnostics tasks it is usually sufficient to store only the values of the coordinate amplitudes of $i_{S x}, i_{S y}, i_{S z}$ vectors.

Fig. 3 gives coordinates of the $i_{S x}$ vector, i.e. the IM response to the $u_{S x}$ impulse voltage action. Curve 1 represents the $i_{A}$ coordinate at the start of IM running. Its amplitude $X$ is taken as one relative unit of current. Curves 2 and 3 represent $i_{A}$ and $i_{C}$ coordinates at some current point in operation. Their amplitudes $X_{A}$ и $x_{C}$ are put down into a Green's matrix, as shown in Fig. 4. The second and third columns of this matrix are filled according to the results of the IM affecting by $u_{S y}$ and $u_{S z}$ vectors. The Green's matrix formed in this way and associated with point of time $t$ has the form:

$$
G(t)=\left(\begin{array}{ccc}
X_{A} & x_{A} & -X_{C} \\
-X_{A} & X_{B} & x_{A} \\
x_{C} & -X_{B} & X_{C}
\end{array}\right)
$$

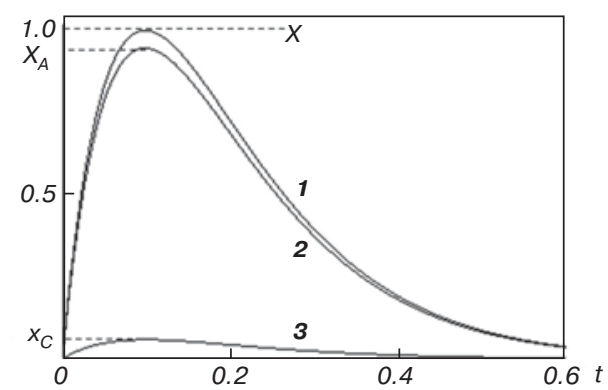

Fig. 3. The IM response to the impulse voltage impact

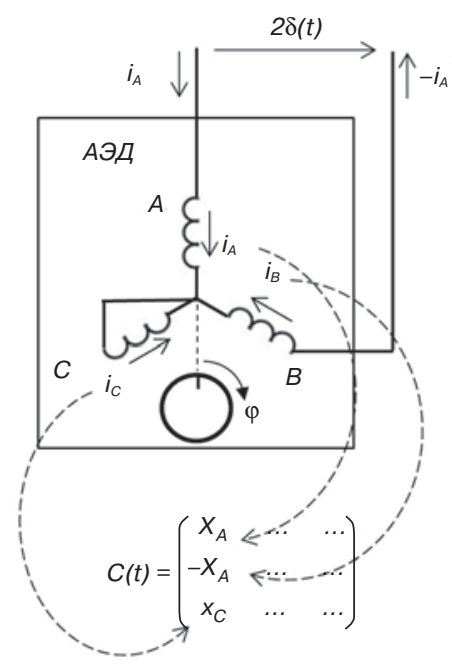

Fig. 4. The formation of a Green's matrix
At the reference technical condition of IM being fixed at the start of operation $t=0$, the Green's matrix has the form:

$$
G(0)=\left(\begin{array}{ccc}
X & 0 & -X \\
-X & X & 0 \\
0 & -X & X
\end{array}\right) .
$$

The matrix of current deviations is formed as the difference

$$
\Delta G(t)=a b s(G(0)-G(t)) .
$$

The matrix of current deviations formed and associated with the current moment of operation $t$ is a medium of objective and reliable information about the IM technical condition.

In the course of operational diagnostics, the matrix of current deviations is compared with the matrix of allowable variations:

$$
\Delta G\left(T_{r}\right)=\left(\begin{array}{ccc}
\Delta X & \Delta x & \Delta X \\
\Delta X & \Delta X & \Delta x \\
\Delta x & \Delta X & \Delta X
\end{array}\right) .
$$

This matrix, which displays the limiting operational state of IM, is formed by an expert method. It should be noted that $G(t), G(0), \Delta G(t)$, and $\Delta G\left(T_{r}\right)$ array cells characterize the device itself and do not depend on the shape and degree of symmetry of the supply voltages. It seems appropriate to store these matrices as a part of the product's in-line documentation for the entire IM life.

Comparison of the matrices of current and limiting deviations allows us to come to a number of conclusions about the IM features.

1. The presence of proportional changes in the matrix elements $\Delta G(t)$ obtained during successive tests $\Delta G\left(t_{1}\right)$, $\Delta G\left(t_{2}\right), \ldots$ indicates a uniform operational aging of the product and its rate.

2. Drastic changes of one of the matrix elements $\Delta G(t)$ testifies that damage has appeared or the nonuniform aging process is in progress.

3. Changing the matrix elements $\Delta G(t)$ when the rotor angular position changes (angle $\varphi$ in Fig. 4) indicates wear of the bearings or damages of rotor IM.

Besides, the comparison of the current and limiting deviation matrices makes it possible to give forecast estimates of the product current probability of survival

$$
p_{0}(t)=1-\max (\Delta G(t))
$$

and the current value of the residual IM resource

$$
T_{r r}=\left(\frac{\Delta X \vee \Delta x}{\max (\Delta G(t))}-1\right) t .
$$

In accordance with expression (3), the maximal element of the current deviation matrix can be interpreted as the probability value of IM failure. Thus, expression (3) combines the approach of Kolmogorov equations and the topological method for analyzing the dynamics of the IM operational changes. 
The information contained in the current deviation matrices has a dual purpose. Firstly, it is meant to substantiate the decisions aimed at improving the efficiency of the IM fleet operation and consisting in on-line replacements, repairs, reservation and varying the load of electric motors. Secondly, it is intended to be used as statistical information to develop criteria for the IM sectoral fleet efficient operation.

On the whole, it can be stated that the approach of Kolmogorov equations and the topological method for analyzing the dynamics of operational changes are effective means of solving the task of enhancing the quality of the electric motor fleet operation in non-ferrous metallurgy.

\section{Expected annual economic effect}

The expected annual economic effect was calculated using the current cost of the branch electric motor fleet $C=16$ billion rubles as the difference between the average annual costs of replacing electric motors:

$$
S\left(T_{c}\right)=\frac{1}{T_{c}} \sum_{t=0}^{T_{c}-1} k_{a} \frac{C}{T_{c}}(1+d)^{t}
$$

at the existing and extended duration of the $T_{c}$ replacement cycle. For the current fleet operation mode, the following values were adopted: $T_{c}=10$ is a cycle duration of planned replacements of electric motors, year, $k_{a}=1.05$ is a coefficient of unscheduled replacements of electric motors, $d$ is an annual rate of increasing the cost, $t$ is current time, year.

For the mode of the fleet efficient operation the following values were taken: $T_{c}=12, k_{a}=1.05$.

The calculation gives an approximate value of the annual economic effect:

$$
E=S(10)-S(12)=2.02-1.72=0.3 \text { billion roubles }
$$
on a sectoral scale.

Thus, application of a sectoral system for IM efficient operation is not only worthwhile, but also profitable.

\section{Conclusions}

In non-ferrous metallurgy, managing the IM fleet efficient operation is a large-scale technical and economic problem, the solution of which will give a significant economic effect.

In scientific terms, the problem is solved in the framework of two research areas: in the direction of developing criteria for the IM sectoral fleet efficient operation and towards elaboration of methods and means for implementing the IM fleet efficient operation.

The basis for developing criteria for efficient operation is modeling the current operational conditions, taking into account the processes of the IM operational aging. The existing methods and models are poorly oriented to fixing changes caused by operational aging, which determines the demand for special methods and aids for modeling the IM operational conditions within the context of the stated problem.
The presented mathematical model based on Kolmogorov equations is one of the means to solve the stated problem for non-ferrous metallurgy or its individual corporations and enterprises.

The system of periodic operational diagnostics is ment to be a key element in enhancement of the IM pool operation efficiency. A topological method worked out for the problems of operational diagnostics is focused on analyzing the dynamics of operational changes taking place in the IM vector space. The matrix of current deviations is a medium of objective and reliable information about the current IM technical condition. Matching the matrices of current and extreme deviations allows us to make several essential conclusions concerning the IM technical state.

Approach of Kolmogorov equations and the topological method for analyzing the dynamics of operational changes are effective means of solving the task to enhance the quality of the electric motor fleet operation in non-ferrous metallurgy.

The reported study was funded under as a part of state assignment (project number, FSWF-2020-0019), as well as at the expense of RFBR (project number, 20-01-00283).

\section{References}

1. Ermakov K. S., Kransutskaya A. A., Tumakova E. V. Measuring Support and Diagnostics of the Operation of Electromechanical Systems Using the Phase-Chronometric Method. Instruments. 2018. No. 5. pp. 33-38.

2. Stepanov V. M., Svistunov N. A. Diagnostics and Management of the Operation Modes of Electromechanical and Electrical Systems with Autonomous Sources of Electrical Energy for Gas Distribution Network. Izvestiya TulGU. Tekhnicheskie nauki. 2018. No. 12. pp. 96-99.

3. Tumakova E. V., Komshin A. S. Development Prospects and Main Problems in the Field of Diagnostics of Electromechanical Systems. The Future of Mechanical Engineering in Russia : Collection of Reports of the Eighth Conference of Young Scientists and Specialists. 2015. pp. 137-140.

4. Shprekher D. M., Kolesnikov E. B. The Remote Method of Diagnosing the Technical Condition of Complex Electromechanical Systems. 2018 International Multi-Conference on Industrial Engineering and Modern Technologies (FarEastCon). DOI: 10.1109/FarEastCon.2018.8602600

5. Ming Yu, Mengxin Li. Fault Detection and Isolation in a Nonlinear Electromechanical System. 2017 International Conference on Sensing, Diagnostics, Prognostics, and Control (SDPC). 2017. DOI: 10.1109/sdpc.2017.58

6. Veresnikov G. S., Skryabin A. V. The Electromechanical Actuator Technical Condition Monitoring System Based on Data Mining Methods. 2018 Eleventh International Conference "Management of Large-Scale System Development" (MLSD). 2018. DOI: $10.1109 / \mathrm{mlsd} .2018 .8551829$

7. Moon J., Leeb S. B. Wire Less Sensors for Electromechanical Systems Diagnostics. IEEE Transactions on Instrumentation and Measurement. 2018. Vol. 67, Iss. 9. pp. 2235- 2246. 
8. Kalugin M. V., Biyukov V. V. Diagnostics and Reliability of Electromechanical Systems of the Transport Complex. Novosibirsk : Izdatelstvo NGTU, 2018. 232 p.

9. Chinmaya B., Galiveeti H. R., Pranju C., Arup K. G., Chandra P. G., Girish K. S. Assessment of Equipment Trip Probability Due to Voltage Sags Based on Fuzzy Possibility Distribution Function. IEEE Access. 2018. Vol. 6. pp. 76889-76899.

10. Miroshin R. N., Barinova O. V. Particular Solutions of Chapman-Kolmogorov Equation And Their Connection With Equatios of Mathematical Physics. Natural and Mathematical Sciences in the Modern World: Proceedings of the XLIV International Scientific-Practical Conference. 2016. Iss. 42, No. 7. pp. 126138.

11. Rahman P. A. Availability Factor of Data Processing System with Primary and Backup Nodes. International Journal of Applied and Fundamental Research. 2015. No. 9. pp. 608611.

12. Kravchik A. E., Shlaf M. M., Afonin V. I., Sobolenskaya E. A. Induction Motors of the 4A Series. Reference Book. Moscow : Energoizdat, 1982. 504 p.
13. Kurilin S. P., Denisov V. N., Fedulov A. S., Dli M. I. Scientific Basis of Methods for Topological Diagnostics of Asynchronous Electric Machines. AIP Conference Proceedings. MECHANICS, RESOURCE AND DIAGNOSTICS OF MATERIALS AND STRUCTURES (MRDMS-2018): Proceedings of the $12^{\text {th }}$ International Conference on Mechanics, Resource and Diagnostics of Materials and Structures. 2018. Vol. 2053. 030031. DOI: $10.1063 / 1.5084392$

14. Kurillin S. P., Denisov V. N., Dli M. I., Bobkov V. I. Vector Space As An Area Of The Operation Risks Characteristics For Asynchronous Electric Machines. Journal of Physics: Conference Series. 2019. Vol. 1260. 052017. DOI: 10.1088/17426596/1260/5/052017

15. Kurilin S. P., Denisov V. N., Dli M. I., Bobkov V. I. A Method for the Operational Diagnostics of Induction Motors. AIP Conference Proceedings. MECHANICS, RESOURCE AND DIAGNOSTICS OF MATERIALS AND STRUCTURES (MRDMS-2019): Proceedings of the $13^{\text {th }}$ International Conference on Mechanics, Resource and Diagnostics of Materials and Structures. 2019. Vol. 2176. 040008. DOI: 10.1063/1.5135157 